\title{
Obtaining Artificially Caries-affected Dentin for in vitro Studies
}

\author{
${ }^{1}$ Cynthia Soares de Azevedo, ${ }^{2}$ Bruna Uglik Garbui, ${ }^{3}$ Caroline Martins e Silva, ${ }^{4}$ Maria Regina Lorenzetti Simionato \\ ${ }^{5}$ Anderson Zanardi de Freitas, ${ }^{6}$ Adriana Bona Matos
}

\begin{abstract}
Aim: This study evaluated and improved a protocol for obtaining standard caries-affected dentin (CAD) by Streptococcus mutans biofilm demineralization process.

Materials and methods: Forty-eight human molars were divided in six experimental groups, according to: period of cariogenic challenge $(7,14$ or 21 days) and type of dentin (erupted or unerupted teeth). After complete cariogenic challenge sound and CAD dentin were evaluated by: visual inspection (VI), digital radiography (DR), optical coherence tomography (OCT) and laser fluorescence (LF).
\end{abstract}

Results: Visual inspection confirmed the formation of CAD based on tissue yellowing and loss of surface gloss. Digital radiography detected the presence of radiolucent images, suggesting caries. Three calibrated examiners viewed all images obtained by $\mathrm{VI}$ and $\mathrm{DR}$ and were able to distinguish healthy from CAD. Fisher's exact statistical test $(p<0.05)$ confirmed no difference between groups by VI (G1/G4: $p=0.6 ; G 2 / G 5: p=1 ; G 3 / G 6$ : $p=1)$ or DR (G1/G4: $p=1 ; G 2 / G 5: p=1 ; G 3 / G 6: p=1)$. Both LF values and demineralization depth, as determined by OCT, were subjected to ANOVA ( $p<0.05$ ). For LF, a statistically significant difference was observed for the type of substrate ( $p$ $=0.001$ ). For OCT, no statistically significant differences in the type of substrate $(p=0.163)$, length of cariogenic challenge $(p=$ $0.512)$ or interaction between factors $(p=0.148)$ were observed. Scanning electron micrographs confirmed the presence of CAD; a more uniform demineralization surface was observed in the dentin of unerupted teeth.

Conclusion: This protocol suggests that standard CAD can be obtained in 7 days of cariogenic challenge using unerupted teeth.

Clinical significance: With the new perspective on the clinical treatment of caries lesions, bonding is increasingly performed to demineralize CAD, which is susceptible to remineralization. A useful protocol to standardize the production of CAD, by

${ }^{1} \mathrm{PhD}$ Student, ${ }^{2,3} \mathrm{BDS},{ }^{4}$ Assistant Teacher, ${ }^{5}$ Researcher and $\mathrm{PhD},{ }^{6}$ Full Professor

${ }^{1-3,6}$ Department of Operative Dentistry, University of São Paulo São Paulo, Brazil

${ }^{4}$ Institute of Biomedical Sciences, University of São Paulo, São Paulo, Brazil

${ }^{5}$ Department of Lasers and Applications, Nuclear and Energy Research, São Paulo, Brazil

Corresponding Author: Adriana Bona Matos, Full Professor Department of Operative Dentistry, University of São Paulo São Paulo, Brazil, e-mail: bona@usp.br microbiological cariogenic challenge, would be an important contribution to laboratorial test in the field of operative dentistry.

Keywords: Dental caries, Diagnosis, Caries-affected dentin, Laboratory research.

How to cite this article: Azevedo CS, Garbui BU, Silva CM, Simionato MRL, Freitas AZ, Matos AB. Obtaining Artificially Caries-affected Dentin for in vitro Studies. J Contemp Dent Pract 2014;15(1):12-19.

Source of support: Grants Fapesp/2010/10126-3 and 2010/ 12910-3.

\section{Conflict of interest: None}

\section{INTRODUCTION}

Regarding to treatment of dental caries, there is no longer any need to completely remove the carious tissue, as was recommended in the past. When caries-affected dentin, namely the dentin underlying the infected dentin, is adequately sealed by restorative material, it is able to reverse the demineralization and harden. ${ }^{1}$ Therefore, this substrate might be kept in the cavity floor, thus avoiding excessive removal of dental tissue and consequently pulp exposure.

Due to its high clinical relevance, studies using naturally affected dentin have been performed, ${ }^{2-9}$ although with controversial results. Authors attributed these results to the lack of standardization of natural carious lesions because of structural differences among different activities of carious process. ${ }^{10,11}$ For this reason, caries-affected dentin produced artificially in a standardized manner might represent an important contribution to in vitro studies.

Models of the artificial formation of carious lesions were developed to improve the understanding of several features of caries lesions, including the preventive effect of fluoride, methods to remove carious tissue, and the adhesive efficacy of restorative materials. ${ }^{12}$ Marquezan et al $^{13}$ studied the main artificial methods used to produce carious dentin, such as acidified gel, $\mathrm{pH}$ cycling, and the microbiological method, and despite the simplicity of the former two, the latter method results in greater similarity in the pattern of collagen degradation compared to natural lesions. Fontana et $\mathrm{al}^{14}$ also cited the possibility of investigating the etiology and prevention of carious lesions, the cariogenic potential of several bacterial species, and the effects of different types of diets as advantages of the bacterial method. 
The studies that have used the microbiological method to produce affected dentin, however, show a lack of methodological standardization. Whereas Zanchi et $a 1^{11}$ established 28 days as the time required for caries induction, other authors ${ }^{15-17}$ chose a 14-day period, and a preliminary study ${ }^{18}$ showed that there is no difference in the depth of demineralization among different times of caries induction (7, 14 and 21 days) when analyzed by means of optical coherence tomography (OCT). However, other and more common methods for caries diagnosis are needed to assess demineralized areas to allow for the reproducibility of results obtained by different laboratories in which OCT is not available. Such diagnostic methods must be able to validate and characterise the investigated substrate without affecting the integrity of specimens.

Some methods are considered classic for the diagnosis of dental caries, including visual inspection complemented by radiographic examination. Radiography has currently evolved to digital systems that allow for the handling of images and the reduction of exposure to ionising radiation. Innovative methods, such as light-induced tissue fluorescence (DIAGNOdent) and OCT, were developed recently, and the last one allows the depth of demineralization in caries lesions to be quantified.

A further factor to take into account regarding standardized substrates is the origin of samples. The teeth employed for research purposes are usually obtained from tooth banks, which do not appropriately record the origin of teeth, their oral status (impacted or erupted), the age of the patient, or the presence of occlusion with the antagonist tooth.

Dentin originating from impacted teeth is quite different from dentin obtained from erupted teeth. ${ }^{19}$ In the latter, there is continuous formation of secondary dentin from the moment of eruption onward, and even mastication and friction might stimulate dentin formation. Therefore, the diameter of the dentinal tubules and the permeability of dentin originating from impacted teeth are greater compared to dentin originating from erupted teeth ${ }^{19}$ subjected to posteruptive maturation.

Although longitudinal and long-lasting studies are ideal, they require too much time before they are concluded and their results can be applied to practice. Therefore, laboratory studies performed with carious or artificially standard induced carious tissue are an important alternative to predict the clinical performance in everyday clinical practice. Thus, this in vitro study aimed to propose a protocol to produce standardized caries-affected dentin in function of time, which was validated by several diagnostic methods.

\section{MATERIALS AND METHODS}

\section{Artificial Occlusal Caries}

Human molar teeth were cleaned with periodontal curettes and a rubber cup with pumice paste and water and were stored in distilled water $\left(4^{\circ} \mathrm{C}\right)$ until use. Approval was obtained from the Ethics Committee (University of São Paulo, Dental School, FOUSP, protocol \#11/2011).

The occlusal surfaces of 48 human molars (24 erupted and 24 unerupted) were abraded with a sandpaper disk until a flat surface was obtained and the occlusal dentin surface was exposed. These teeth were sectioned in half in the buccal-lingual direction, and the 84 resulting halves were distributed among six experimental groups $(n=14)$, according to the type of substrate (erupted ou unerupted) and length of cariogenic challenge (7, 14 or 21 days), as follows: G1-erupted +7 days; G2-erupted +14 days; G3-erupted + 21days; G4-unerupted +7 days; G5-unerupted +14 days; G6-unerupted +21 days.

Half the area of the prepared teeth was covered with acid-resistant nail varnish (Colorama, CEIL Coml Exp Ind Ltd., São Paulo, SP, Brazil), and the sample was sterilized with gamma irradiation ( $25 \mathrm{kGy}$ ). Caries lesions were then induced in vitro with the use of a bacterial system as described below. ${ }^{20}$

Cultures of Streptococcus mutans (UA 159) were incubated in Tryptic Soy Broth medium (TSB, Difco, Becton, Dickinson and Company, Sparks, MD, USA), supplemented with $5 \%$ sucrose $\left(37^{\circ} \mathrm{C}, 24\right.$ hours), to obtain bacterial growth. Bacteria were spread on the surface of Tryptic Soy Agar (TSA, Difco, Becton, Dickinson and Company) solid culture medium to obtain isolated colonies, which were then re-incubated $\left(37^{\circ} \mathrm{C}, 48\right.$ hours). After growth, the colonies were transferred into tubes containing TSB (with $5 \%$ sucrose), and the dental halves of the same group were immersed in a solution $(300 \mathrm{ml}$ of TSB with $5 \%$ sucrose and $3 \mathrm{ml}$ of inoculum broth) and were maintained therein for 7 (groups G1 and G4), 14 (groups G2 and G5), or 21 (groups G3 and G6) days. Each dental half was transferred to a fresh tube every 24 hours. During the incubation periods, tests were performed to check for the presence of bacterial contaminants, and the dental halves were then removed from the bacterial system after the specified times, according to experimental groups.

At the end of the cariogenic challenge, the biofilm adhering to the surface was carefully removed, and the specimens were washed with deionized water. Immediately before the demineralization depth was read, the acid-resistant nail varnish was mechanically removed from the surfaces of all specimens.

\section{OCT Imaging}

\section{System Configuration}

In the most common OCT systems, a Michelson interferometer is used to provide a cross-sectional image of the scattering specimens, with noninvasive evaluation and 
without contact with their surfaces. In this system, the light is conducted by an optical fiber to a $2 \times 2$ coupler, where the light is split into two beams, one of which is sent to a reference mirror and the other to the sample.

In the present OCT system (SR930 Thorlabs Inc., Newton, NJ, USA), a super-luminescent light-emitting diode $(\lambda=930 \mathrm{~nm})$ provided A and B scans with 4.0 and $6.0 \mu \mathrm{m}$ resolution, respectively. The system was able to produce up to four frames per second, thus providing real-time images $(2000 \times 512$ pixels; equivalent to a width $\times$ height area of $\left.6000 \times 1581 \mu^{2}\right)$.

The images were acquired from the central regions of the exposed windows, generating images from both exposed and varnish-protected (control) surfaces. The images of the caries-affected dentin were obtained by OCT for different exposure periods (7, 14 and 21 days), and different dentin substrates (from erupted and unerupted teeth) were compared according to demineralization depth. If any tissue loss occurred due to the demineralization process, the amount of lost tissue was included in the calculation, with the adjacent sound dentin used as the initial reference.

\section{Examination with Laser Fluorescence}

The DIAGNOdent unit was calibrated prior to each use according to the manufacturer's instructions, using the instrument's ceramic standard. When measurements were being made on teeth, the instrument was zeroed with the tip in contact with healthy dentin. ${ }^{21}$ Examination was performed on both the control (healthy) and affected dentin surfaces to obtain individualized data from each fragment. Next, the tip of the probe (indicated for fissures and smooth surfaces) was placed perpendicular to the demineralized dentin area. Each area was measured three times as described above, and the result for each specimen was calculated from the mean of the obtained fluorescence measurements.

\section{Digital Radiography}

Images were obtained by means of a digital interproximal radiographic technique using advanced digital imaging (Dabi Atlante, São Paulo, SP, Brazil) with an X-ray device at $70 \mathrm{kVp}$ and $1 \mathrm{~mA}$. Specimens were standardly positioned, the focus-detector distance was $21 \mathrm{~cm}$, and the captured images were directly digitalized and displayed by the system software (ProImage, Elmsford, NY, USA). The images were inspected on a well-illuminated computer screen by three calibrated examiners who were asked to distinguish between healthy and caries-affected dentin.

\section{Visual Inspection}

Visual inspection was performed under appropriate artificial illumination and after drying with a air spray for
5 seconds. Artificially produced CAD and the adjacent sound dentin were recorded by means of standardized photographs using a video camera coupled to the OCT; all such images were taken from the same distance and subsequently described based on their qualitative features. The images were inspected on a well-illuminated computer screen by three calibrated examiners who were asked to distinguish between healthy and caries-affected dentin.

\section{Morphological Analysis}

Immediately after caries induction, two samples from each group were prepared for dentin surface analysis by scanning electron microscopy (SEM). The samples were immersed in $2.5 \%$ glutaraldehyde for 24 hours at $4{ }^{\circ} \mathrm{C}$ for fixation and then washed in distilled water three times for 1 minute. For dehydration, the specimens were sequentially immersed in ethanol (30\% for 10 minutes, $50 \%$ for 10 minutes, $70 \%$ for 10 minutes, $90 \%$ for 10 minutes, $96 \%$ for 10 minutes and $100 \%$ for 20 minutes) and then transferred to a critical-point dryer (HMDS) for 30 minutes. The prepared specimens were mounted on aluminum stubs, gold-sputtered at $10 \mathrm{~mA}$ for 1 minute and analyzed under SEM.

\section{Statistical Analysis}

Numerical data obtained during assessment with laser fluorescence (DIAGNOdent) and from the measurement of the depth of dentinal demineralization (in $\mu \mathrm{m}$ ) by means of OCT were subjected to statistical analysis using two-way analysis of variance (ANOVA) with a 5\% significance level. The data obtained from digital radiography and visual inspection by three calibrated examiners were subjected to Fisher's exact test at the 5\% significance level to verify the differences between the obtained accuracy rates in percentage.

\section{RESULTS}

\section{Quantitative Analysis}

\section{Laser Fluorescence}

The results obtained from laser fluorescence were significantly affected by the type of substrate $(p=0.001)$. However, the length of cariogenic challenge $(p=0.132)$ and the interaction between factors $(p=0.616)$ did not have significant effects on the results. These results show that the groups containing fragments from erupted teeth (G1, G2 and G3) exhibited higher average values of LF when compared to fragments from impacted teeth (G4, G5 and G6).

\section{OCT}

The results of OCT did not show significant effects of the type of substrate $(p=0.163)$, the length of cariogenic challenge $(p=0.512)$, or the interaction between factors $(\mathrm{p}=0.148)$ on the results. 


\section{Visual Inspection and Digital Radiography}

The examiners distinguished caries-affected and healthy dentin in all experimental groups with accuracy rates ranging from 78.5 to $100 \%$ (visual inspection) and from 92.8 to $100 \%$ (digital radiography).

There was no statistically significant difference among groups when compared based on either visual inspection [G1 and G4 $(\mathrm{p}=0.6), \mathrm{G} 2$ and $\mathrm{G} 5(\mathrm{p}=1)$ and G3 and G6 $(\mathrm{p}=1)]$ or digital radiography $[\mathrm{G} 1$ and $\mathrm{G} 4(\mathrm{p}=1), \mathrm{G} 2$ and G5 $(\mathrm{p}=1)$ and $\mathrm{G} 3$ and $\mathrm{G} 6(\mathrm{p}=1)]$.

\section{Qualitative Analysis}

\section{Visual Inspection and Digital Radiography}

Figure 1 shows a representative photograph used for visual inspection and a representative image taken using digital radiography. Healthy dentin was distinguished from cariesaffected dentin in all experimental groups.

Visual inspection detected changes in color and surface shine, e.g. caries-affected dentin was more yellow and opaque than healthy dentin. Digital radiography revealed a radiolucent area corresponding to demineralized tissue as well as a mild loss of structure (yellow arrow) (Fig. 1).

\section{OCT}

Optical coherence tomography can generate three types of images, namely traditional (similar to the images generated by ultrasound), inverse, and color (demineralized areas appear in hues of grey or black on a white background), and all three types were obtained from the 84 specimens. Figure 2 illustrates these three types of images generated by OCT from dental fragments subjected to cariogenic challenge; the vertical bar distinguishes between demineralized (right side) and healthy (left side) dentinal surfaces. OCT established the depth of demineralization in both types of substrates at the investigated lengths of demineralization, limited by arrows in the images. This depth was similar among all experimental groups, and mild loss of structure was observed in all investigated specimens.

\section{SEM}

One image of each group $(\times 200$ magnification $)$ was chosen to illustrate the morphology of demineralized dentin (Figs $3 \mathrm{~A}$ to F). After 7 days of cariogenic challenge in impacted teeth characteristic CAD was observed (D). This dentin could be described as an irregular surface with preserved peaks and valleys and opened dentinal tubules on the entire image. The fissure seen in $\mathrm{D}$ appears to be a drying artefact. On the other hand, images $\mathrm{A}, \mathrm{B}$ and $\mathrm{E}$ showed a regular pattern of demineralization where peaks and valleys could not be detected, without features of CAD. Images $\mathrm{C}$ and $\mathrm{F}$ showed opened dentinal tubules in a smooth entire surface indicating an intense acid demineralization due to the longer length of cariogenic challenge and thus, loss of CAD characteristics.

Sound dentin from all experimental groups is represented in Figure 4 ( $\times 200$ magnification). Dentin is completely covered by smear layer, with grooves produced by grounding procedure.

\section{DISCUSSION}

Caries-affected dentin obtained in vitro by the microbiological method brings an important contribution to adhesion knowledge of dental materials, because it will allow laboratory tests to be performed on a standard caries-affected dentin which is a clinically relevant substrate. ${ }^{7,22-24}$ The microbiological method allowed us to artificially produce standard caries-affected dentin in 7 days, using impacted teeth.

Using the photomicrographs generated by SEM, we compared the dentinal substrates derived from impacted and erupted teeth at different length of cariogenic challenge. We found more uniform superficial demineralization across the entire extent of caries-affected dentin derived from impacted teeth, where only primary dentin is formed. Moreover, it seems that a longer cariogenic challenge promotes an intense demineralization which removes the irregularity feature of CAD.

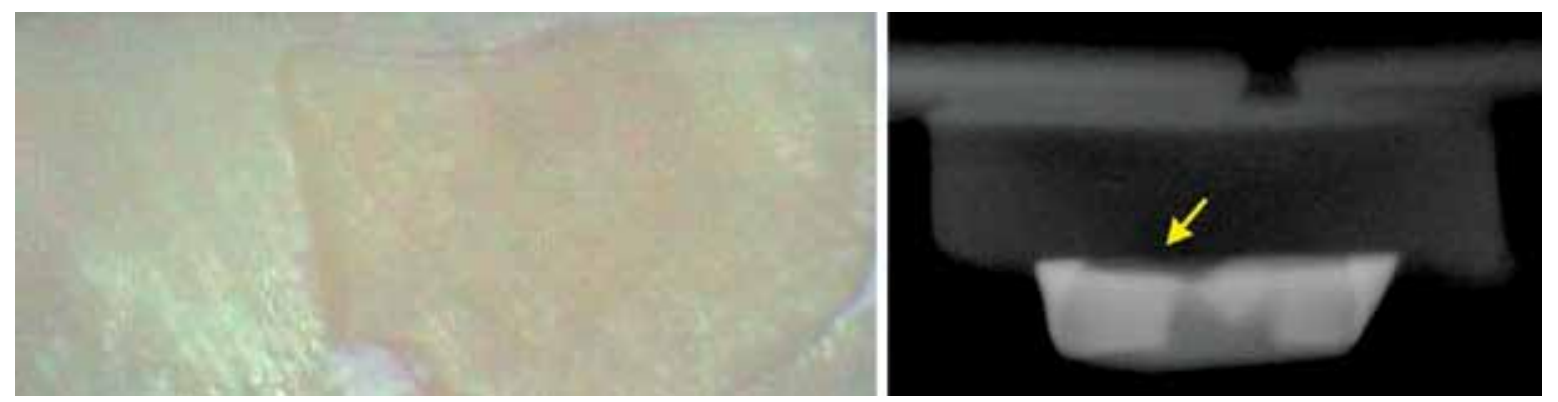

Fig. 1: In the photograph (left), the left side of the image is compatible with healthy dentin and the right side of the image is compatible with caries-affected dentin, which exhibits less shine and a yellower surface compared to healthy dentin. In the digital radiograph (right), the yellow arrow points to the border between healthy and affected dentin. Note the patent loss of tissue to the left of the arrow, corresponding to the area of caries-affected dentin. In this same area, there is radiolucency characteristic of demineralization 


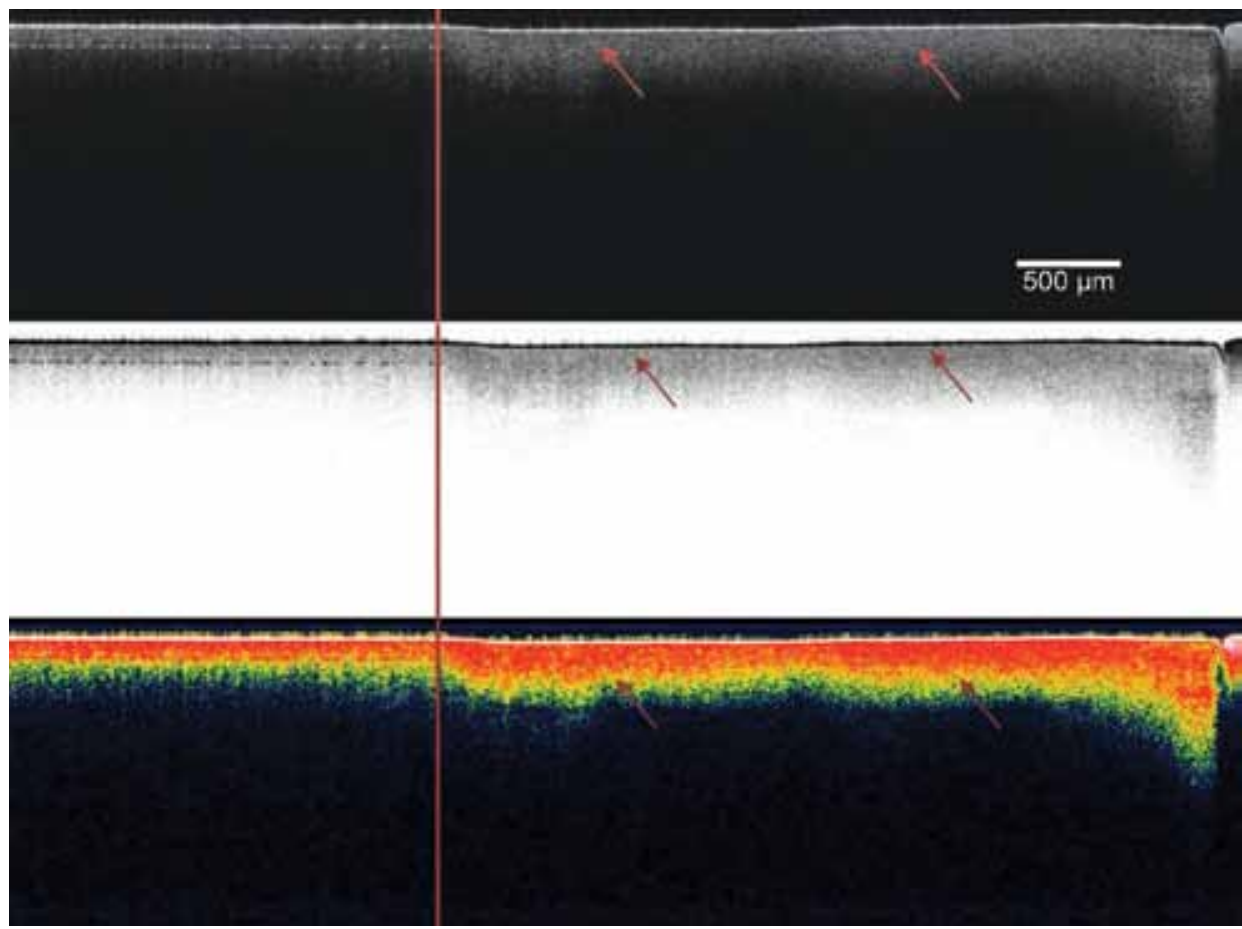

Fig. 2: Representative images obtained with OCT in a single specimen. The vertical bar separates healthy from artificial caries-affected dentin. Arrows point to the demineralized area of caries-affected dentin
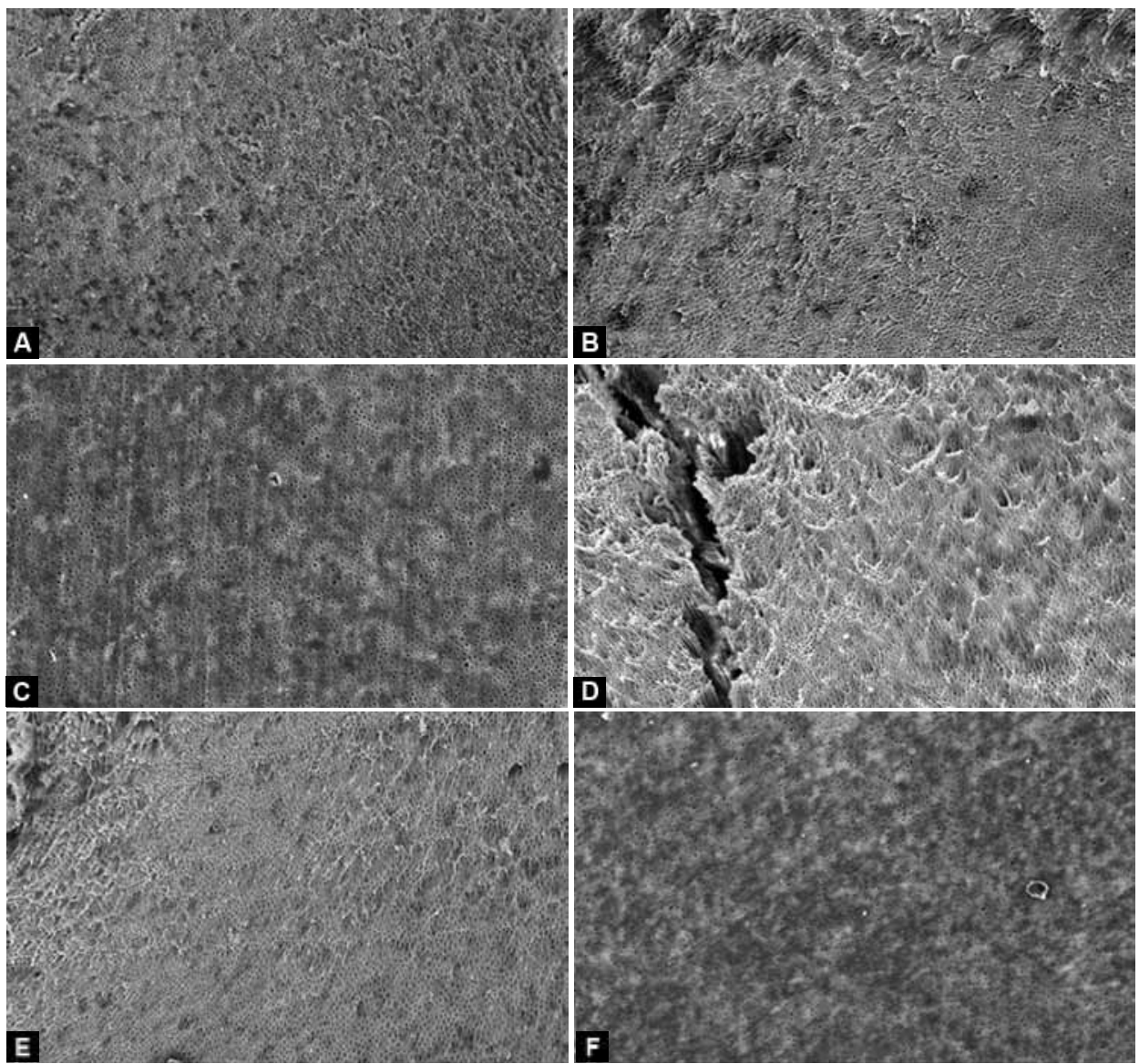

Figs 3A to F: Scanning electron micrograph of CAD of all experimental groups ( $\times 200$ magnification): (A) group G1 (specimens subjected to microbiological cariogenic challenge for 7 days, substrate derived from erupted tooth), (B) group G2 (specimens subjected to microbiological cariogenic challenge for 14 days, substrate derived from erupted tooth), (C) group G3 (specimens subjected to microbiological cariogenic challenge for 21 days, substrate derived from erupted tooth), (D) group G4 (specimens subjected to microbiological cariogenic challenge for 7 days, substrate derived from impacted tooth), (E) group G5 (specimens subjected to microbiological cariogenic challenge for 14 days, substrate derived from impacted tooth), (F) group G6 (specimens subjected to microbiological cariogenic challenge for 21 days, substrate derived from impacted tooth) 


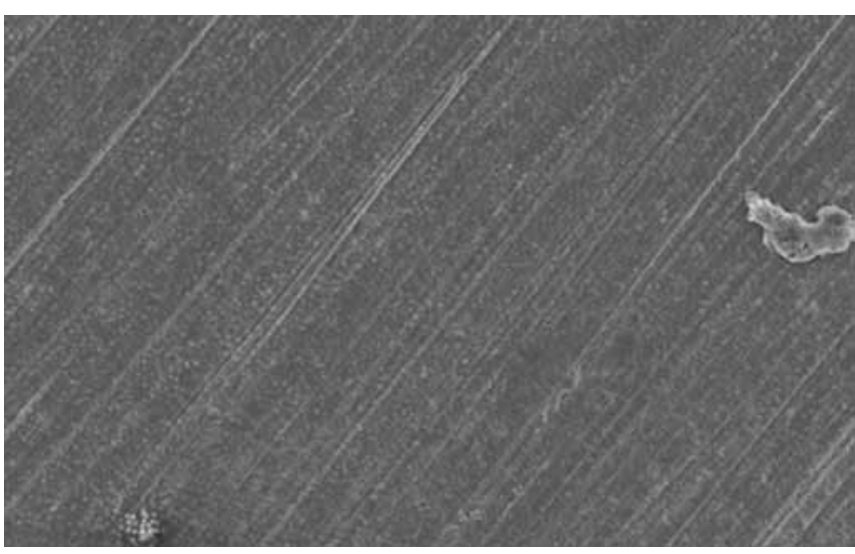

Fig. 4: Scanning electron microscopy of sound dentin $(\times 200$ magnification). Dentin is completely covered by smear layer with grooves produced by grounding procedure

Dentin derived from impacted teeth differs from dentin originating from erupted teeth, ${ }^{1}$ because they undergo different challenges, such as intrinsic processes, like production of secondary dentin from the moment of their eruption into the oral cavity, exposure to extrinsic processes, such as friction caused by mastication and carious processes that induce the production of reparative dentin. ${ }^{1}$ Therefore, from the morphological perspective, differences in the tubular diameter might be observed with increased deposition of peritubular dentin, which is the product of the maturation process. ${ }^{19}$ For this reason, dentinal permeability decreases, however this process does not occur simultaneously across the entire dentinal surface. Thus, the dentin derived from erupted teeth should exhibit greater heterogeneity compared to the dentin originating from impacted teeth, which might directly interfere with the results of laboratorial essays, for example, bond strength tests. This fact was emphasized by Mjör et al, ${ }^{25}$ who called attention to the care required from researchers when selecting the dental elements constituting the study samples.

The four diagnostic methods used in the present study, visual inspection, digital radiography, laser fluorescence and optical coherence tomography, were effective in distinguishing between healthy and artificial caries-affected substrates at all investigated time points (7,14 and 21 days).

Visual inspection, a valuable diagnostic method for both in vitro ${ }^{26-28}$ and in vivo ${ }^{29,30}$ applications, produced good results in the distinction between artificial CAD and sound dentin with high level of agreement between the three examiners.

Digital radiography is highly recommended as an auxiliary diagnostic examination together with visual inspection. In this study, we included inter-examiner analyses ${ }^{31}$ to increase the accuracy of our results. Although digital radiography results present their limitations in the early identification of images suggestive of caries lesions, our results show that, for the level of demineralization to which the specimens were subjected, all three calibrated examiners identified dentinal radiolucency in the cariesaffected areas in all experimental groups.

Laser fluorescence produces good efficient results in the detection of both occlusal and interproximal caries lesions for both in vivo and in vitro studies. ${ }^{27,29,32-35}$ It is worth noting that among the results obtained with laser fluorescence, a significant difference was observed between impacted and erupted teeth. Dental fragments derived from erupted teeth exhibited greater fluorescence in caries-affected dentin compared to dentin derived from impacted teeth. This difference most likely occurred because dentin from erupted teeth was more pigmented than the dentin of impacted teeth, as previously observed. ${ }^{35}$

We chose to use visual inspection, digital radiography and laser fluorescence together, not only due to its good results, but because they are available in all teaching institutions, dental offices as well as research laboratories and they can effectively establish the presence or absence of carious tissue.

OCT, a novel technology with promising results in the detection of caries lesions, ${ }^{18,36,37}$ can also detect and quantify the depth of demineralization produced without destroying the specimen. ${ }^{38}$ The images clearly show that demineralization occurred inside the specimens at all different times of exposure to bacterial challenge, without exhibiting significant differences among the three cariogenic challenge time points.

Freitas et $\mathrm{al}^{39}$ assessed demineralization induced by a bacterial strain for up to 11 days and found an exponential increase in the depth of demineralization up to 9 days. Those authors suggested that this increase was correlated with the exhaustion of nutrients in the culture, the accumulation of inhibitory metabolites and final products, or a lack of biological space. Their results might also agree with ours because the depth of demineralization was not different on days 7, 14 and 21. We believe that the nutrient-exhaustion hypothesis does not apply to the conditions of our study because nutrients were supplied every 24 hours, when the culture medium was replaced. However, the accumulation of metabolites and final products, as well as the lack of biological space, might indeed explain the lack of progression of the depth of demineralization after 7 days.

The above findings might be explained as follows. In vivo, the biofilm that naturally accumulates on the surface of teeth is a complex community comprising more than 500 bacterial species, ${ }^{40,41}$ whereas the first colonisers are mostly streptococci. At the onset of heterogeneous bacterial interactions, signalling might play an important role, whereas the adhesion and coaggregation of different microorganisms contribute to the maturation of the biofilm. In the presence of saccharose, the enzyme glucosyltransferase catalyzes the 
production of glucans out of water-insoluble polysaccharides that might favor colonization by $S$. mutans. ${ }^{42}$ The initial colonising bacteria most likely generate an environment favorable to new invaders, thereby facilitating the survival of other species that are better able to adapt to the new habitat and that eventually replace the initial species. The biofilm in the present study comprised only colonies of S. mutans and thus lacked any competitive or inhibitory substance produced by other species. In addition, the bacterial deposition on the cariogenic challenge days gave rise to a thick biofilm. Although we did not measure biomass, our results show that the depth of demineralization was maintained between days 7 and 21, which might be explained as a function of the thick layer of bacterial deposition formed during caries induction. Above all, this was not considered a problem for us, because our main goal was to obtain caries-affected dentin to be used in our laboratory adhesion studies, and not obtaining a complete caries lesion with all its distinct carious zones.

The lack of differences by all tested diagnostic methods in the three time points of our study indicates that a period of 7 days is sufficient to obtain standardized affected dentin for use in laboratory assays. This conclusion is relevant for two main reasons: (1) the microbiological method used to obtain caries lesions is quite sensitive and requires trained researchers; thus, the odds of succeeding in the in vivo production of this type of substrate with no risks of contamination are higher when using a significantly shorter time and, (2) bond strength tests performed with naturally affected dentin produced divergent results, e.g. some studies found lower adhesion values in caries-affected compared to healthy dentin, ${ }^{3,7-9}$ whereas other studies did not find statistically significant differences between the two investigated substrates. ${ }^{2,45}$ Therefore, this standard protocol for the production of caries-affected dentin in the laboratory will facilitate the performance of in vitro studies where the substrate will no longer influence the investigated parameters.

\section{CONCLUSION}

The present protocol suggests that caries-affected dentin can be artificially produced using the microbiological method of cariogenic challenge for 7 days, preferentially in impacted teeth.

\section{CLINICAL SIGNIFICANCE}

With the new perspective on the clinical treatment of caries lesions, bonding is increasingly performed to demineralize $\mathrm{CAD}$, which is susceptible to remineralization. A useful protocol to standardize the production of CAD, by microbiological cariogenic challenge, would be an important contribution to laboratorial test in the field of operative dentistry.

\section{REFERENCES}

1. Fejerskov OKE, Dentária C. A doença e seu tratamento clínico. São Paulo: Editora Santos, 2011.

2. Ergucu Z, Celik EU, Unlu N, Turkun M, Ozer F. Effect of Er,Cr:YSGG laser on the microtensile bond strength of two different adhesives to the sound and caries-affected dentin. Oper Dent 2009 Jul-Aug;34(4):460-466.

3. Komori PC, Pashley DH, Tjaderhane L, Breschi L, Mazzoni A, de Goes MF, et al. Effect of $2 \%$ chlorhexidine digluconate on the bond strength to normal versus caries-affected dentin. Oper Dent 2009 Mar-Apr;34(2):157-165.

4. Mobarak EH. Effect of chlorhexidine pretreatment on bond strength durability of caries-affected dentin over 2-year aging in artificial saliva and under simulated intrapulpal pressure. Oper Dent 2011 Nov-Dec;36(6):649-660.

5. Mobarak EH, El-Badrawy W, Pashley DH, Jamjoom H. Effect of pretest storage conditions of extracted teeth on their dentin bond strengths. J Pros Dent 2010 Aug;104(2):92-97.

6. Mobarak EH, El-Korashy DI, Pashley DH. Effect of chlorhexidine concentrations on micro-shear bond strength of self-etch adhesive to normal and caries-affected dentin. Am J Dent 2010 Aug;23(4):217-222.

7. Pereira PN, Nunes MF, Miguez PA, Swift EJ Jr. Bond strengths of a one-step self-etching system to caries-affected and normal dentin. Oper Dent 2006 Nov-Dec;31(6):677-681.

8. Scholtanus JD, Purwanta K, Dogan N, Kleverlaan CJ, Feilzer AJ. Microtensile bond strength of three simplified adhesive systems to caries-affected dentin. J Adhes Dent 2010 Aug;12(4): 273-278.

9. Wang L, Sakai VT, Kawai ES, Buzalaf MA, Atta MT. Effect of adhesive systems associated with resin-modified glass ionomer cements. J Oral Rehabil 2006 Feb;33(2):110-116.

10. Pugach MK, Strother J, Darling CL, Fried D, Gansky SA, Marshall SJ, et al. Dentin Caries Zones: Mineral, Structure and Properties. J Dent Resear 2009 Jan;88(1):71-76.

11. Zanchi CH, Lund RG, Perrone LR, Ribeiro GA, del Pino FA, Pinto MB, et al. Microtensile bond strength of two-step etchand-rinse adhesive systems on sound and artificial caries-affected dentin. Am J Dent 2010 Jun;23(3):152-156.

12. Joves GJ, Inoue G, Nakashima S, Sadr A, Nikaido T, Tagami J. Mineral density, morphology and bond strength of natural versus artificial caries-affected dentin. Dent Mater J 2013;32(1): 138-143.

13. Marquezan M, Correa FNP, Sanabe ME, Rodrigues LE, Hebling J, Guedes-Pinto AC, et al. Artificial methods of dentin caries induction: a hardness and morphological comparative study. Archives of Oral Biology 2009 Dec;54(12):1111-1117.

14. Fontana M, Dunipace AJ, Gregory RL, Noblitt TW, Li Y, Park $\mathrm{KK}$, et al. An in vitro microbial model for studying secondary caries formation. Caries Res 1996;30(2):112-118.

15. Espejo LC, Simionato MR, Barroso LP, Netto NG, Luz MA. Evaluation of three different adhesive systems using a bacterial method to develop secondary caries in vitro. Am J Dent 2010 Apr;23(2):93-97.

16. Marquezan M, Osorio R, Ciamponi AL, Toledano M. Resistance to degradation of bonded restorations to simulated cariesaffected primary dentin. Am J Dent 2010 Feb;23(1):47-52.

17. Sanabe ME, Costa CA, Hebling J. Exposed collagen in aged resin-dentin bonds produced on sound and caries-affected dentin in the presence of chlorhexidine. J Adhes Dent 2011 Apr;13(2):117-124. 
18. Azevedo CS, Trung LC, Simionato MR, Freitas AZ, Matos AB. Evaluation of caries-affected dentin with optical coherence tomography. Braz Oral Res 2011 Sep-Oct;25(5):407-413.

19. Costa LR, Watanabe IS, Kronka MC, Silva MC. Structure and microstructure of coronary dentin in non-erupted human deciduous incisor teeth. Braz Dent J 2002;13(3):170-174.

20. Gama-Teixeira A, Simionato MR, Elian SN, Sobral MA, Luz MA. Streptococcus mutans induced secondary caries adjacent to glass ionomer cement, composite resin and amalgam restorations in vitro. Braz Oral Res 2007 Oct-Dec;21(4):368-374.

21. Markowitz K, Stenvall RM, Graye M. The effect of distance and tooth structure on laser fluorescence caries detection. Oper Dent 2012 Mar-Apr;37(2):150-160.

22. Azevedo CS, Matos AB. Laboratorial method proposal to obtain caries-affected dentin. Saarbrucken/Germany: LAP Lambert Academic Publisher; 2013.

23. Carvalho FG, Goncalves LS, Carlo HL, Soares CJ, CorrerSobrinho L, Puppin-Rontani RM. Influence of sterilization method on the bond strength of caries-affected dentin. Braz Oral Res 2009 Jan-Mar;23(1):11-16.

24. Perdigao J. Dentin bonding-variables related to the clinical situation and the substrate treatment. Dental Materials 2010 Feb;26(2):E24-E37.

25. Mjor IA, Nordahl I. The density and branching of dentinal tubules in human teeth. Arch Oral Biol 1996 May;41(5):401-412.

26. Jablonski-Momeni A, Liebegall F, Stoll R, Heinzel-Gutenbrunner M, Pieper K. Performance of a new fluorescence camera for detection of occlusal caries in vitro. Lasers Med Sci 2012 Mar 21.

27. Neuhaus KW, Rodrigues JA, Hug I, Stich H, Lussi A. Performance of laser fluorescence devices, visual and radiographic examination for the detection of occlusal caries in primary molars. Clin Oral Investig 2011 Oct;15(5):635-641.

28. Novaes TF, Matos R, Gimenez T, Braga MM, Mendes FM. Performance of fluorescence-based and conventional methods of occlusal caries detection in primary molars - an in vitro study. Int J Paediatr Dent 2012 Nov;22(6):459-466.

29. Chen J, Qin M, Ma W, Ge L. A clinical study of a laser fluorescence device for the detection of approximal caries in primary molars. Int J Paediatr Dent 2012 Mar;22(2):132-138.

30. Novaes TF, Matos R, Braga MM, Imparato JCP, Raggio DP, Mendes FM. Performance of a pen-type laser fluorescence device and conventional methods in detecting approximal caries lesions in primary teeth — in vivo study. Caries Research 2009;43(1): $36-42$.

31. Nytun RB, Raadal M, Espelid I. Diagnosis of dentin involvement in occlusal caries based on visual and radiographic examination of the teeth. Scandinavian J Dent Resear 1992 Jun;100(3):144-148.

32. Chen J, Qi M. A study of a laser fluorescence device for assessing caries removal in primary teeth in vitro. Hua Xi Kou Qiang Yi Xue Za Zhi 2011 Oct;29(5):457-460.

33. de Paula AB, Campos JA, Diniz MB, Hebling J, Rodrigues JA. In situ and in vitro comparison of laser fluorescence with visual inspection in detecting occlusal caries lesions. Lasers Med Sci 2011 Jan;26(1):1-5.

34. Rodrigues JA, Diniz MB, Josgrilberg EB, Cordeiro RC. In vitro comparison of laser fluorescence performance with visual examination for detection of occlusal caries in permanent and primary molars. Lasers Med Sci 2009 Jul;24(4):501-506.

35. Rodrigues JA, Hug I, Diniz MB, Lussi A. Performance of fluorescence methods, radiographic examination and ICDAS II on occlusal surfaces in vitro. Caries Res 2008;42(4):297-304.

36. Can AM, Darling CL, Ho C, Fried D. Nondestructive assessment of inhibition of demineralization in dental enamel irradiated by a lambda $=9.3$ microm $\mathrm{CO}_{2}$ laser at ablative irradiation intensities with PS-OCT. Lasers in Surgery and Medicine 2008 Jul;40(5):342-349.

37. Lee C, Darling CL, Fried D. Polarization-sensitive optical coherence tomographic imaging of artificial demineralization on exposed surfaces of tooth roots. Dental Materials 2009 Jun;25(6):721-728.

38. Gimbel C. Optical coherence tomography diagnostic imaging. General Dentistry 2008 Nov;56(7):750-757.

39. Freitas AZ, Zezell DM, Mayer MPA, Ribeiro AC, Gomes ASL, Vieira ND. Determination of dental decay rates with optical coherence tomography. Laser Physics Letters 2009 Dec; 6(12):896-900.

40. Kolenbrander PE, Andersen RN, Blehert DS, Egland PG, Foster JS, Palmer RJ. Communication among oral bacteria. Microbiology and Molecular Biology Reviews 2002 Sep;66(3):486.

41. Paster BJ, Boches SK, Galvin JL, Ericson RE, Lau CN, Levanos $\mathrm{VA}$, et al. Bacterial diversity in human subgingival plaque. $\mathrm{J}$ Bacteriol 2001 Jun;183(12):3770-3783.

42. Banas JA, Vickerman MM. Glucan-binding proteins of the oral streptococci. Crit Rev Oral Biol Med 2003;14(2):89-99.0007 